\title{
Strukturpolitik in der Krise - Mehr als Standort- wettbewerb und Innovationsförderung
}

Astrid Zieg/er

\begin{abstract}
Aktuell wird nicht nur weltweit über Konjunkturprogramme diskutiert, sie werden auch in großer Zahl und mit vor wenigen Monaten kaum vorstellbaren finanziellen Mitteln umgesetzt. Einen Baustein stellen dabei Hilfspakete für Unternehmen dar, die infolge der Finanz- und Konjunkturkrise in Schwierigkeiten geraten sind. Damit gerät ein Politikfeld - die Strukturpolitik - in den Mittelpunkt öffentlicher Debatten, das sonst eher stiefmütterlich behandelt wird. Der Staat hilft Unternehmen schon seit Jahrzehnten mit milliardenschweren Programmen; die Qualität der Arbeit und beschäftigungspolitische Ziele sollten jedoch stärker in den Fokus rücken.
\end{abstract}

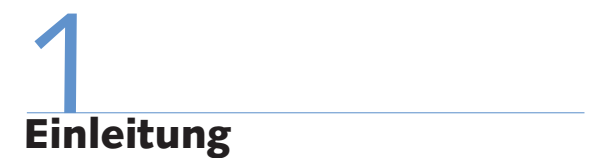

In Deutschland wird schon seit einigen Jahrzehnten Strukturförderung von Bund und Bundesländern betrieben. Diese nationalen und länderspezifischen Förderstrategien stehen nicht selten in Konkurrenz zueinander. Die Strukturförderung des Bundes zielt allgemein darauf ab, die Wettbewerbs- und Innovationsfähigkeit der deutschen Wirtschaft zu verbessern. Die Strukturpolitik der Länder beruht auf regionalpolitischen Vorgaben unter Berücksichtigung der landesspezifischen Rahmenbedingungen. Ein zunehmend wichtiger finanzieller und politischer Rahmen sind in den letzten Jahren die Europäischen Strukturfonds geworden.

Im Prinzip verfolgte die staatliche Strukturpolitik lange Zeit das Ziel, die Lebensbedingungen in den Regionen anzugleichen und die ökonomische und soziale Spaltung in unserer Gesellschaft zu vermindern. Obwohl nach Evaluierungsstudien diese ausgleichspolitische Strukturförderung durchaus erfolgreich war, geriet sie im Zuge von Globalisierung und auferlegtem öffentlichen Sparzwang unter Druck. In den letzten Jahren hat sich daher die Stoßrichtung der Strukturpolitik in Deutschland grundlegend verändert. Sie bewegt sich heute zwischen Standortwettbewerb und Innovationsförderung. Prägend ist nämlich der Wettbewerb um die innovativsten Unternehmen, die attraktivsten Standorte und die besten Fachkräfte. In dieser Gemengelage spielen beschäftigungspolitische Aspekte kaum noch eine Rolle. Angesichts der derzeitigen Konjunktur- und Strukturkrise ist damit fraglich, ob alleine der Ausbau be- stehender Instrumente - wie derzeit im Konjunkturpaket I und II geschehen - die in den nächsten Wochen und Monaten auf uns zukommenden Arbeitsmarktprobleme bewältigen kann. Oder ob nicht doch ein grundlegender Politikwechsel beim Einsatz strukturpolitischer Instrumente notwendig wäre. Denn gerade in beschäftigungspolitisch schwierigen Zeiten kann und darf sich staatliches Handeln nicht selektiv nur um die vermeintlich Starken kümmern und nach Maßstäben einer betriebswirtschaftlichen Markt- und Wettbewerbsstrategie handeln. Da neue Arbeitsplätze per Saldo nicht so schnell entstehen wie alte wegbrechen, sind staatliche Aktivitäten als volkswirtschaftlich notwendig und nützlich anzusehen, die den stattfindenden Strukturwandel an traditionellen Industriestandorten nachhaltig, d.h. ökonomisch erfolgreich und sozial-ökologisch ausgerichtet, gestalten und generell Beschäftigung auf dem ersten Arbeitsmarkt sichern. Zu diesem Zweck muss der Staat sich strukturpolitische Kompetenzen wieder aneignen.

Im Folgenden gebe ich zunächst einen Überblick über die aktuellen Trends der wirtschaftlichen Entwicklung (Abschnitt 2), skizziere anschließend, welche Auswirkungen die wirtschaftliche Entwicklung auf die Beschäftigten hat (Abschnitt 3) und zeige exemplarisch an der betrieblichen Unternehmensförderung und der Umorientierung strukturpolitischer Inhalte und Akteure, welche untergeordnete Rolle mittlerweile das Thema Beschäftigung spielt (Abschnitt 4). Der Beitrag schließt mit der Forderung nach einer Neuausrichtung der Förderpolitik (Abschnitt 5).

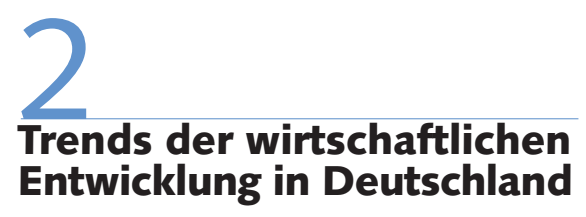

Die deutsche Wirtschaft befindet sich in einer tiefen konjunkturellen Krise. Finanzmarktkrise und Konjunktureinbruch verstärken sich wechselseitig - so schreibt das Institut für Makroökonomie und Konjunkturforschung (IMK) in seinem letzten Konjunkturbericht (IMK 2009). Zwar stieg das reale Bruttoinlandsprodukt (BIP) im Jahresdurchschnitt 2008 immerhin um $1,3 \%$, aber diese noch günstige Entwicklung ist auf das erste Quartal 2008 zurückzuführen. Im Jahresverlauf ist das BIP indes um 1,4 \% geschrumpft - im vierten Quartal dürfte es einen beträchtlichen Rückgang von saisonbereinigt 1,8\% gegeben haben. Verantwortlich für diese Entwicklung ist vor allem der Exporteinbruch infolge der stark rückläufigen Auftragseingänge aus dem Ausland, insbesondere bei den Investitionsgütern. Damit gehen eine sinkende Kapazitätsauslastung und deutlich schlechtere Finanzierungsbedingungen für betriebliche Investitionen einher. Die Konjunkturkrise trifft in weiten Teilen unserer Wirtschaft mit einer Strukturkrise zusammen, die sich - je nach Branche - in eine Absatz- und Produktkrise weiter ausdifferenziert. Besonders dramatisch zeigt sich dies momentan in der Automobilindustrie, Teilen des Maschinenbaus und der Chemischen Industrie. 
Auch ohne Konjunkturkrise gibt es in Volkswirtschaften Größenverschiebungen zwischen und innerhalb von volkswirtschaftlichen Teilbereichen. Ursachen dieses Strukturwandels können prinzipiell drei Gruppen - angebotsseitige, nachfrageseitige und institutionelle-zugeordnet werden (Meißner/Fassing 1989, S. 60ff.). Sie bestimmen in ihrem Zusammenwirken die langfristigen strukturellen Entwicklungen der Volkswirtschaft und ihrer Teilbereiche. Kennzeichen der langfristigen wirtschaftlichen Entwicklung der letzten Jahre sind in Deutschland grob drei Trends:

(1) Einer der wesentlichen Trends ist die Verflechtung von Industrie und Dienstleistungen. Die Wertschöpfung erfolgt in zunehmendem Maße in Wertschöpfungsketten über verschiedene Branchen und Sektoren hinweg und nicht mehr vorrangig innerhalb traditionell abgegrenzter Branchen. So ist z. B. der Industriesektor heutiger Prägung ein enger Verbund von traditioneller industrieller Produktion und industrienahen Dienstleistungen. Zwar wird heute der weit überwiegende Teil des Bruttoinlandsprodukts im Dienstleistungssektor erwirtschaftet, aber die Wachstumsdynamik in diesem Sektor wird maßgeblich von der Nachfrage nach unternehmensbezogenen und industrienahen Dienstleistungen gespeist, die ihrerseits von der Industrieproduktion und der Endnachfrage nach industriellen Produkten abhängt. Die gesamtwirtschaftliche Bedeutung einer Branche kann deswegen nicht mehr nur durch die Zahl der dortigen Arbeitsplätze gemessen werden. Der Blick muss auf die gesamte Wertschöpfungskette - im Falle der Industrie auf den gesamten Industrie-Dienstleistungsverbund gerichtet werden. Und: Der industrielle Sektor hat nach wie vor eine hohe Relevanz für Produktion und Beschäftigung in Deutschland, wobei die besonders exportorientierten Industrien (der sogenannte Export-Kern) eine hervorgehobene Stellung einnehmen (Lichtblau/Ziegler 1991; Kalmbach et al. 2005).

(2) Die wirtschaftliche Entwicklung und insbesondere die Unternehmensentwicklung sind durch eine forcierte Internationalisierung und dabei aus deutscher Sicht vor allem durch eine Europäisierung gekennzeichnet. Einerseits betrifft dies sowohl Handels- und Kapitalströme als auch Unternehmensstrukturen und Unterneh- mensstandorte. So haben Auslandsinvestitionen deutscher Unternehmen, speziell in europäische Nachbarländer, und eine zunehmende grenzüberschreitende Kapitalverflechtung zur Herausbildung europäisch bzw. weltweit agierender Konzerne geführt. Deutsche Unternehmen nutzen die Chancen offener Märkte für Investitionen und zusätzliche Produktion. Sie verlagern Unternehmensteile ins Ausland oder gliedern sie aus. Sie bauen neue Standorte im Ausland auf. All dies führt dazu, dass die Standortkonkurrenz innerhalb von Unternehmen und Konzernen zunimmt. Ein klassisches Beispiel dafür ist die Automobilindustrie, die transnationale Produktionsnetze rund um den Globus aufgebaut hat (Blöcker 2009). Wie das Beispiel Opel gerade anschaulich zeigt, erweisen sich diese internationalisierten Wirtschaftsbeziehungen im Zuge der Konjunkturkrise im konkreten Einzelfall als schwere Bürde.

Andererseits sind auch wirtschaftsund industriepolitische Kompetenzen in den vergangenen Jahrzehnten zunehmend auf die europäische Ebene verlagert worden. Wesentliche Regelungen wie z. B. die Beihilfe- und Wettbewerbskontrolle, Rahmenbedingungen der Strukturpolitik, internationale Abkommen und Außenhandelsregulierungen, Umweltvorschriften und anderes mehr werden mittlerweile auf europäischer Ebene entschieden. Für Konjunkturprogramme bedeutet dies beispielsweise, dass die europäischen Mitgliedstaaten in den letzten Wochen ihre Programme bei der EU-Kommission genehmigen lassen mussten. Die EU-Kommission überprüft ihrerseits, ob dadurch europäische Wettbewerbsbestimmungen verletzt werden. ${ }^{1}$

(3) In Deutschland wurde die wirtschaftliche Entwicklung zusätzlich seit Beginn der 1990er Jahre von der deutschen Vereinigung beeinflusst. Die Bilanz des Aufbaus Ost fällt gemischt aus. Einige Regionen leiden immer noch an den Folgen der massiven De-Industrialisierung, die im Zuge der Wiedervereinigung einsetzte. An anderen Standorten gelang es jedoch, Kerne einer wettbewerbsfähigen und neuen Wirtschaftsstruktur mit starken Branchenakzenten zu etablieren (Jakszentis/Hilpert 2005; Rosenfeld et al. 2006). Als Folge dieses Umstrukturierungsprozesses konzentrieren sich die staatlichen Hilfen der Wirtschaftsförderung auf Ostdeutschland, auch im Jahr 20 der Wiedervereinigung liegen die wirtschaftlichen Schwerpunkte vorrangig in den westlichen Bundesländern.

Aufgrund dieser Trends schreitet der nationale, regionale und sektorale Standortwettbewerb auf internationaler Ebene wie innerhalb Deutschlands unverändert voran.

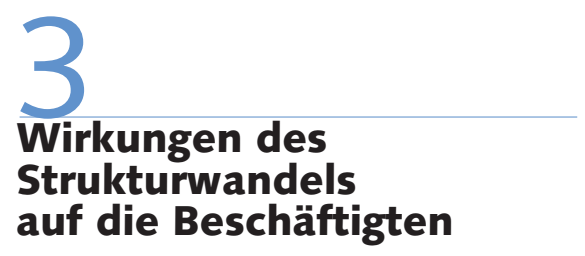

Was bedeutet nun diese wirtschaftliche Entwicklung für die Beschäftigten? Um diese Frage zu beantworten, muss die aktuelle Situation auf dem Arbeitsmarkt von der Entwicklung vor der Krise unterschieden werden:

Die aktuelle Konjunkturkrise ist mittlerweile am Arbeitsmarkt angekommen. Noch weist die Statistik einen moderaten Anstieg der Arbeitslosenzahlen aus, aber die Hiobsbotschaften über geplante Entlassungen mehren sich. Derzeit nutzen Betriebe massiv das beschäftigungspolitische Instrument der Kurzarbeit, um Zeit zu gewinnen, kurzfristig Kosten zu senken und sich nicht durch voreilige Entlassungen substanziell zu schaden (Seifert 2009).

Aber auch schon in wirtschaftlich weniger turbulenten Zeiten hat der Strukturwandel in vielen Situationen die Risiken für die Beschäftigten verschärft. Nach der WSIBetriebsrätebefragung von 2007 nutzte z. B. ein nicht unerheblicher Teil der Arbeitgeber betriebliche Umstrukturierungsmaßnahmen wie Verlagerung oder Ausgliederung, um sowohl am bisherigen Standort als auch im verlagerten und ausgegliederten Betrieb Druck auf die Beschäftigten auszuüben und die Löhne zu senken oder die Arbeitszeiten auszuweiten (Ziegler 2008). Wie die Zahlen der Betriebsrätebefragung zeigen, hatten zwar Verlagerungen und Ausgliederungen am bisherigen Standort in gut der Hälfte der Betriebe keine Auswirkungen auf die Arbeitsbedingungen. In einem Teil der Betriebe mit Standortverlagerungs- und Ausgliederungserfahrung schätzten die Be-

\footnotetext{
1 Vgl. z. B. die deutschen Bürgschaftsregeln (http://ec. europa.eu/deutschland/press/pr_releases/8316_ de.htm)
} 
triebsräte die Arbeitsbedingungen am bisherigen Standort sogar besser ein als vor der Maßnahme. Dies machten sie daran fest, dass nach der durchgeführten Maßnahme die Arbeitsplätze sicherer geworden sind oder beispielsweise die Arbeitsorganisation optimiert wurde. Trotzdem gab es einen nicht zu vernachlässigenden Anteil an Betrieben, in denen sich die Arbeitsbedingungen an den bisherigen Standorten negativ verändert hatten: Für die Beschäftigten war der Arbeitsdruck angestiegen, das Betriebsklima hatte sich verschlechtert und die Beschäftigten mussten nach der Umstrukturierungsmaßnahme mehr und länger arbeiten als vorher. Trotz Umstrukturierung sind die verbliebenen Arbeitsplätze unsicherer geworden. Dieses Bild findet sich auch in den verlagerten Betrieben, allerdings in etwas anderen Größenordnungen. Am negativsten stellten sich die Auswirkungen auf die Arbeitsbedingungen in den ausgegliederten Betrieben dar. Fast jeder zweite Betriebsrat aus den ehemaligen Betrieben gab an, dass sich dort die Arbeitsbedingungen für die Beschäftigten verschärft hatten (ebd.).

Auf der einen Seite schließen in Deutschland viele Betriebe im Laufe eines Jahres oder verlagern ihre Standorte ins Ausland bzw. gliedern Funktionsbereiche aus. Auf der anderen Seite entstehen auch häufig im Jahresverlauf neue Betriebe. Daneben prägen Branchenkrisen wie die der Textilindustrie, des Bergbaus, der Fotoapparateproduktion, der Automobilindustrie oder der Aufbau neuer Branchen, wie z. B. die Solar- und Windkraftindustrie, die Informations- und Kommunikationstechnik (IuK), die Nano- und Biobranche, den wirtschaftlichen Strukturwandel. Neue Arbeitsplätze entstehen per Saldo allerdings nicht so schnell wie alte wegbrechen, während die Strukturen innerhalb der Gesamtwirtschaft gleichzeitig heterogener werden. Diese Entwicklungen haben nicht nur Auswirkungen auf die Beschäftigten, sondern oft auch auf den jeweiligen Standort und das regionale Umfeld. Für Regionen können betriebliche Umstrukturierungsprozesse oder der Niedergang ganzer Branchen erhebliche Auswirkungen auf die wirtschaftliche Strukturentwicklung haben und eine Abwärtsspirale in Gang setzen: Der Unternehmensbestand schrumpft, es werden weniger Arbeitsplätze zur Verfügung gestellt und der regionale Arbeitsmarkt wird kleiner, tendenziell geht die Nachfrage nach Dienstleistungen und Gütern zurück, die kommunalen Steuereinnahmen sinken, die gut ausgebildeten Bevölkerungsschichten wandern ab. Für die Region steigt das Risiko, dass die Infrastruktur nicht mehr ausgelastet und die Versorgung der Bevölkerung mit grundlegenden Dienstleistungen wie Bildung, Gesundheit und Gütern des täglichen Bedarfs nicht mehr gewährleistet werden kann.

\section{1 \\ Strukturpolitische Antworten}

Richtung und Tempo des wirtschaftlichen Strukturwandels folgen keineswegs unveränderbaren Vorgaben, sondern sie werden beeinflusst durch wirtschafts- und gesellschaftspolitische Zielvorstellungen. Bund und Bundesländer unterstützen die deutsche Wirtschaft mit vielfältigen staatlichen Maßnahmen. Die Strukturförderung will dabei einerseits die Wettbewerbs- und Innovationsfähigkeit der deutschen Wirtschaft stärken und andererseits Beschäftigung sichern und schaffen. Wie der vorherige Abschnitt aber zeigt, haben sich die Risiken für die Beschäftigten eher vergrößert.

Dieser Aspekt des wirtschaftlichen Strukturwandels wird in der Strukturpolitik nicht thematisiert. Ganz im Gegenteil: Seit Mitte der 1990er Jahre ist ein grundlegender Wandel von Zielen, Instrumenten und Inhalten strukturpolitischer Aktivität zu erkennen. Dieses Umsteuern wird deutlich mit den Megatrends der 1990er Jahre in Zusammenhang gebracht: Vereinigung der beiden deutschen Staaten, die europäische Integration (Binnenmarkt, Wirtschafts- und Währungsunion, Osterweiterung), Einbeziehung der ehemaligen RGW-Staaten in die internationale Arbeitsteilung sowie zunehmende Internationalisierung der Wirtschaftsbeziehungen (Ziegler 2006). Die Diskussion um die Folgen dieser Megatrends bei einer gleichzeitig in Deutschland ausbleibenden Verbesserung von Wachstum und Arbeitsmarkt stellte die Effizienz und Effektivität der Strukturpolitik infrage bzw. erforderte ihre Neuausrichtung. Mit diesen Problemen stand Deutschland nicht allein. Auch auf EUEbene mehrten sich die Stimmen, die im Sinne der Lissabon-Strategie Strukturpolitik in Europa mit reduziertem Finanzvolumen unter wachstumsorientierten Aspek- ten einsetzen und auf Wachstumszentren konzentrieren wollen.

Wie allerdings die Evaluierung von staatlichen Maßnahmen der Wirtschaftsförderung zeigte, deckte sich die öffentliche Wahrnehmung über den Misserfolg strukturpolitischer Instrumente nicht mit den tatsächlichen Wirkungen entsprechender Aktivitäten. Sowohl makroökonomische Studien als auch einzelbetriebliche Wirkungsanalysen bestätigen z. B. der Investitionsförderung positive Effekte auf Wachstum und Beschäftigung. So zeigt die makroökonomische Evaluierung der Investitionsförderung (Gemeinschaftsaufgabe Verbesserung der regionalen Wirtschaftsstruktur (GRW), steuerliche Investitionszulage) in der Studie von Schalk/Untiedt (2000) für das Verarbeitende Gewerbe in 327 westdeutschen Kreisen auf Basis eines dynamischen Panelmodells positive, signifikante Nettoeffekte auf Investitionen, Wertschöpfung und Erwerbstätigkeit. Auch Unternehmensbefragungen (u.a. GEFRA et al. 2004) bescheinigen der Investitionsförderung eine positive Wirkung. Koller (2004) belegt anhand des Matching-Ansatzes am Beispiel der GRW, dass geförderte Betriebe eine bessere wirtschaftliche Performance als die Gesamtheit der Betriebe haben; in Ostdeutschland sind die Unterschiede zwischen geförderten Betrieben und den anderen Betrieben sogar noch stärker ausgeprägt.

Ungeachtet dieser Ergebnisse fand ein Umsteuern in der Strukturpolitik statt. Aus Beschäftigtensicht hat die Strukturpolitik in den letzten Jahren allerdings eine falsche Richtung eingeschlagen, denn beschäftigungsrelevante Aspekte sind zunehmend in den Hintergrund getreten. Angesichts der beschäftigungspolitisch schwierigen Zeiten, die vor uns liegen, kann diese Entwicklung in den kommenden Monaten sehr ungünstige Rückwirkungen haben.

\subsection{BETRIEBLICHE FÖRDERPOLITIK}

Ein wesentlicher Baustein zur Bewältigung des Strukturwandels ist die betriebliche Unternehmensförderung. Diese wird in Deutschland zwar im engen Zusammenhang mit der Gemeinschaftsaufgabe „Verbesserung der regionalen Wirtschaftsstruktur" diskutiert, zur betrieblichen Förderung können aber im weiteren Sinne auch Instrumente der Innovations- und Technologiepolitik, der Mittelstands- und Existenzgründungsförderung oder auch der Ener- 
Abb. 1: Staatliche Beihilfen* in den EU-Mitgliedsländern 2007 - in \% des Bruttoinlandprodukts (BIP) -

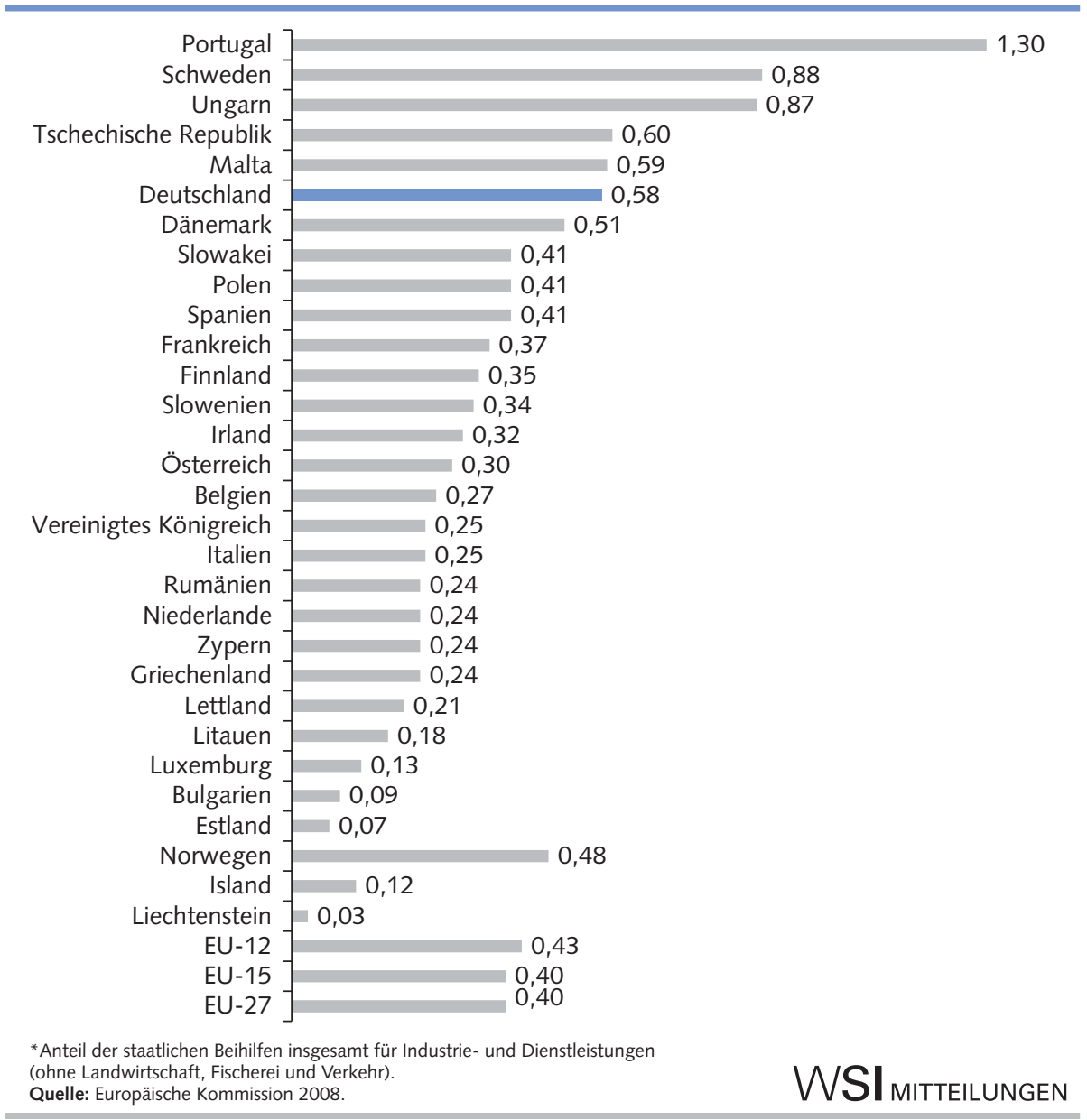

giepolitik gezählt werden, weil auch diese darauf ausgerichtet sind, Unternehmen in ihrer Entwicklung zu helfen. Es gibt eine kaum überschaubare Programmvielfalt der Wirtschaftsförderung von Bund und Bundesländern (www.foerderdatenbank.de). Trotzdem lassen sich einige Grundlinien im Förderkonzept erkennen. In Abhängigkeit von der Zielstellung werden die Programme klassischerweise in vier Kategorien eingeteilt. Es geht um die Förderung von betrieblichen Investitionen, um Existenzgründungen, um die Förderung von Forschung und Entwicklung oder auch um Krisenbewältigung. Der Schwerpunkt der Wirtschaftsförderung wurde in den letzten Jahren vor allem auf Innovation und Mittelstandsförderung ausgerichtet. Kriseninstrumente spielten bis vor kurzem keine nennenswerte Rolle.

Für die betriebliche Unternehmensförderung geben Bund und Bundesländer jährlich mehrere Milliarden Euro aus. Nach dem neuesten Beihilfeanzeiger der Europäischen Kommission flossen in Deutsch-
25 Mrd. €. Ein Unternehmen, das eine Bürgschaft oder einen Kredit beantragt, muss volkswirtschaftlich besonders förderungswürdig sein. Es kann beispielsweise einer Schlüsselbranche angehören oder für die Region besonders wichtig sein (http: //www.bundesregierung.de/Webs/Breg/ konjunkturpaket/DE/Unternehmenskredite/ unternehmenskredite.html - 13.03.2009).

Weitere große und wichtige Förderinstrumente, die aktuell in Deutschland eingesetzt werden, um die Wettbewerbsund Innovationsfähigkeit deutscher Betriebe zu erhöhen, sind:

(1) Europäische Strukturfonds: Sie bilden das wichtigste Instrument der Förderung ökonomischer und sozialer Kohäsion und Solidarität in der Europäischen Union (EU). Sie sollen zu einer ausgewogenen Entwicklung in der EU führen, regionale und soziale Ungleichheiten abbauen sowie die Lissabon-Strategie und die Europäische Beschäftigungsstrategie unterstützen. Zwischen 2007 und 2013 erhält Deutschland über die Europäischen Strukturfonds ca. 26 Mrd. $€$ (Ziegler 2008).

(2) Hightech-Strategie: Die Bundesregierung hat 2006 ressortübergreifend alle Anstrengungen der Innovationspolitik des Bundes in der Hightech-Strategie gebündelt. Damit will Deutschland bis zum Jahr 2010 das Lissabon-Ziel erreichen und den Anteil von Forschung und Entwicklung auf $3 \%$ des Bruttoinlandsproduktes (BIP) erhöhen. Über die Hightech-Strategie werden bis 2009 rund 15 Mrd. $€$ für Forschung, Technologie und Innovation bereitgestellt (Ziegler 2007).

(3) Gemeinschaftsaufgabe, Verbesserung der regionalen Wirtschaftsstruktur" (GRW): Die Mittel der GRW bilden nach wie vor das Rückgrat der Wirtschaftsförderung in Deutschland. Die Investitionsförderung im Rahmen der GRW zielt darauf ab, strukturelle Probleme in Betrieben zu überwinden und wettbewerbsfähige Dauerarbeitsplätze zu schaffen und zu sichern. Gleichwohl nimmt sich die Finanzausstattung gemessen an den anderen Fördertöpfen eher bescheiden aus. So wurden 2008 für die Gemeinschaftsaufgabe „Verbesserung der regionalen Wirtschaftsstruktur" 644 Mio. $€$ vorgesehen. Dieser Betrag wurde im Rahmen des Konjunkturprogramms I um 200 Mio. $€$ aufgestockt (http://www. bmwi.de/BMWi/Navigation/Wirtschaft/ 
Wirtschaftspolitik/Regionalpolitik/gemein schaftsaufgabe.html-04.03.2009).

Ein großer Teil dieser Mittel fließt direkt an Unternehmen. Wie Abbildung 2 zeigt, spielen für die Betriebe die Zuschüsse für Investitionen und Sachmittel eine geringere Rolle als die Förderung der Lohn- und Gehaltskosten. Die ausgewiesenen Förderquoten stellen allerdings bei der Investitionsförderung lediglich die Untergrenze der tatsächlichen Inanspruchnahme öffentlicher Mittel durch die Betriebe dar, weil das IAB-Betriebspanel nur Zuschüsse, nicht aber zinsgünstige Kredite, Zulagen oder Bürgschaften erfasst. Gleichwohl erreichte die Inanspruchnahme der öffentlichen Förderung bei beiden Förderarten Mitte der 1990er Jahre ihren Höhepunkt, in den letzten Jahren ist sie stetig zurückgegangen. Am aktuellen Rand erhielten trotzdem noch $9 \%$ der ostdeutschen Betriebe und $4 \%$ der westdeutschen Betriebe öffentliche Zuschüsse für Investitionen und Sachmittel, bei den Lohnkosten lag die Förderquote bei $13 \%$ in Ostdeutschland und $8 \%$ in Westdeutschland.

Diese Daten sagen aber nichts über die Höhe der gezahlten Zuschüsse aus und auch nichts darüber, wieviele Arbeitsplätze und vor allem welche Arten von Arbeitsplätzen über die öffentliche Förderung gesichert und geschaffen werden konnten.

Welche Auflagen müssen die Betriebe erfüllen, um in den Genuss der Mittel zu kommen? Betriebe erhalten die Förderung nur, wenn sie auf Basis eines schlüssigen Konzeptes nachweisen können, dass die zur Förderung stehende Investition ihre Wettbewerbsfähigkeit stärkt und zu Beschäftigung führt. Dafür muss der Betrieb einen spürbaren Eigenbeitrag übernehmen. Das geförderte Unternehmen muss in der Regel nachweisen, dass es eigene Investitionen tätigt und Arbeitsplätze sichert oder schafft. Die Anzahl der zu schaffenden bzw. zu sichernden Arbeitsplätze ist grob von der Investitionssumme abhängig. Generell können wir feststellen, dass es nur noch wenige Programme gibt, die die Förderung nicht an die Schaffung und Sicherung von Arbeitsplätzen koppelt. Da das Kriterium Arbeitsplätze aber nicht das Hauptziel der Unternehmensförderung ist, werden nicht selten die ursprünglichen Arbeitsplatzzusagen nach unten korrigiert. Bei der Mittelvergabe spielen qualitative Aspekte der geförderten Arbeitsplätze keine Rolle, wie

\section{Abb. 2: Inanspruchnahme öffentlicher Zuschüsse* für ...}

\section{... Investitionen und Sachmittel}

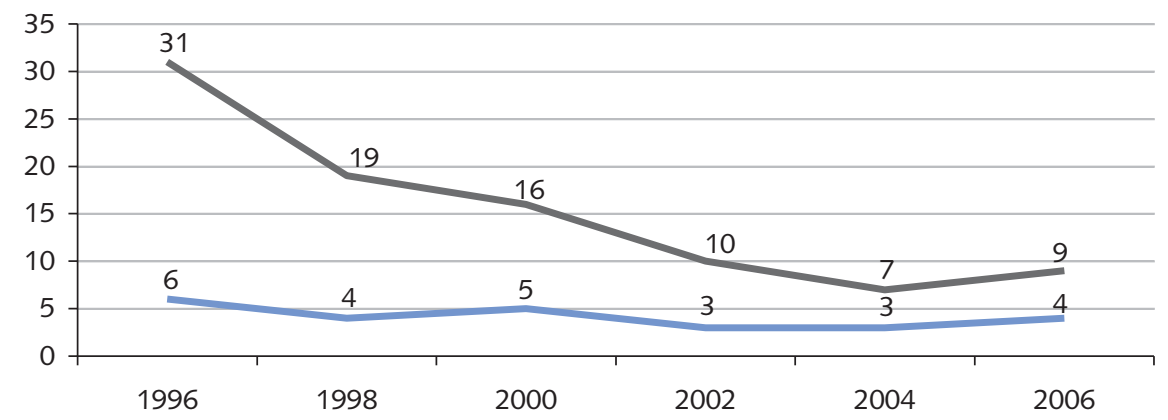

... Lohnkosten

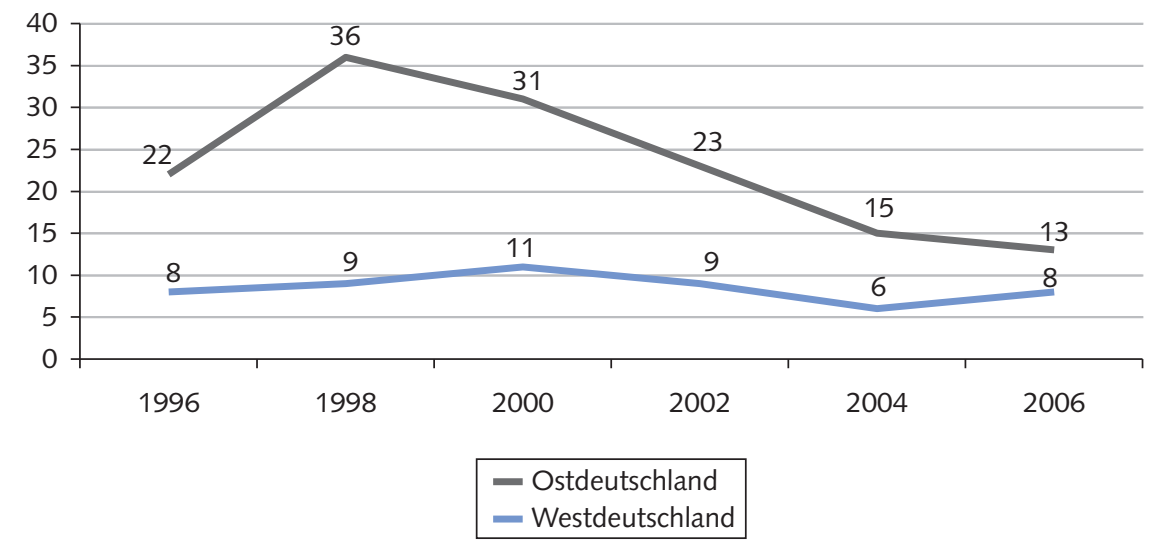

*Anteil der geförderten Betriebe in Ost- und Westdeutschland 1996-2006 in \%. Quelle: Wahse, J./Dahms, V. (2008, S. 58 ff.); Darstellung der Autorin. MITTEILUNGEN

z. B. die Höhe des Einkommens, die Arbeitsbedingungen oder ob der geförderte Betrieb die Wahl eines Betriebsrats behindert.

Am Beispiel der GRW wird im Folgenden verdeutlicht, welche beschäftigungspolitischen Auflagen den Betrieben gemacht werden und wie unterschiedlich zum Teil die Vorgaben sind.

Generell unterliegen alle Förderprogramme in Deutschland dem EU-Beihilferecht. Das Beihilferecht setzt den Rahmen für u. a. die Abgrenzung der Förderinhalte und -sätze sowie die Art der förderfähigen Kosten. In Bezug auf die GRW legen Bund und Bundesländer innerhalb des EU-Beihilferahmens gemeinsam die Regelungen für die Förderung fest. Die Länder sind alleine für die Durchführung zuständig und können ihre Förderrichtlinien strenger ausgestalten als es der Koordinierungsrahmen vorsieht.

Die GRW fördert sachkapitalbezogene Kosten und Lohnkosten. Nach dem Rah- menplan der Gemeinschaftsaufgabe setzt die Gewährung von lohnkostenbezogenen Investitionszuschüssen voraus, dass durch die Investition Arbeitsplätze mit überdurchschnittlicher Qualifikationsanforderung, Arbeitsplätze mit besonders hoher Wertschöpfung oder Arbeitsplätze in Bereichen mit besonders hohem Innovationspotenzial entstehen. Zwar schreibt der Rahmenplan feste Obergrenzen für die Fördersumme in Relation zu den geschaffenen bzw. gesicherten Dauerarbeitsplätzen vor. Nach aktuell geltenden Regeln beträgt der Förderhöchstsatz je geschaffenem Dauerarbeitsplatz $500.000 €$ und $250.000 €$ je gesichertem Dauerarbeitsplatz, um die Förderung bei besonders kapitalintensiven und weniger arbeitsplatzschaffenden Investitionen zu begrenzen. Dafür muss sich das geförderte Unternehmen lediglich innerhalb eines Zeitraums von fünf Jahren ab dem Zeitpunkt der Gewährung der Hilfe verpflichten, die zugesagten Arbeitsplätze an dem betreffenden 
halten zu können. Viele Beispiele zeigen, dass angesichts mächtiger Konkurrenz vielmehr ein gemeinsames, abgestimmtes Vorgehen von vielen Regionen und Betrieben etwa bei Innovation, Marketing, Qualifizierung und Nutzung von Arbeitskräften und Maschinen ein besserer Weg sein kann. Wie die Erfahrungen mit betrieblichen Umstrukturierungen in Ostdeutschland zudem zeigen, lassen sich auch gefährdete Regionen und Branchen sowie Unternehmen unter verschärften Weltmarktbedingungen in Deutschland mit geeigneten politischen Instrumenten stabilisieren.

(3) Räumlich geht es heute um Wirtschaftsregionen und insbesondere um solche Standorte, die einen großen Beitrag zu Wachstum und Innovation leisten. Eine zentrale Rolle spielen die Metropolregionen, die gestärkt werden müssen, weil sie als Motoren der wirtschaftlichen, sozialen und kulturellen Entwicklung gelten. Hier werden die Wachstums- und Innovationspotenziale gesehen, um Deutschland international wettbewerbs- und konkurrenzfähig zu machen (Müller/Sträter 2008, S.270ff.). Aber diesen Wirtschaftsregionen fehlt als Akteur oder Institution nicht selten demokratische Legitimation und Transparenz.

(4) Organisatorisch geht es um die Kooperation und Vernetzung von Unternehmen und Wissenschaft. Dazu wird in zunehmendem Maß das Instrument des Wettbewerbs eingesetzt. Ziel dieser Wettbewerbe ist es, die Kooperation und Vernetzung verschiedener Akteure zu intensivieren, die an einem gemeinsamen Projekt arbeiten. Die einzelnen Projekte stehen in Konkurrenz zueinander, das Land entscheidet nach bestimmten Kriterien, welches Projekt gefördert wird. In diesen Wettbewerben werden die Akteure aufgefordert, innovative Projektideen in Projektteams gemeinsam zu entwickeln und sich bei der entsprechenden Stelle zu bewerben. Über diesen Weg sollen innovative Verbundprojekte identifiziert und anschließend die besten Projekte gefördert werden.

Zusammenfassend ist festzustellen, dass heute die Strukturpolitik von wissensintensiven Unternehmen, Forschungs- und Entwicklungseinrichtungen dominiert wird. Außen vor bleiben Arbeitnehmerinteressen und -vertretungen, soziale und nicht-wett- bewerbsorientierte Politikbereiche. Das heißt, die neue Strukturpolitik ist in dem Sinne wettbewerbsorientiert, dass sie nach dem Motto „Starke stärken“ eindeutig auf Hightech und Leuchttürme, Zukunftsbranchen, Wettbewerbe sowie Hochqualifizierte setzt. Zwar ist gegen Wettbewerb und Innovationsförderung generell nichts zu sagen. Nur wenn man - wie in der aktuellen Strukturpolitik - die Akzente einseitig setzt, schüttet man gewissermaßen das Kind mit dem Bade aus. Die Strukturpolitik ist selektiv geworden und schließt die vermeintlich Schwachen aus, wie Un- und Angelernte, Branchen des Lowtech-Bereichs, strukturschwache Regionen etc.

Eine solch einseitige Ausrichtung auf sogenannte Leuchttürme vernachlässigt außerdem, dass einige wenige HightechUnternehmen allein nicht hinreichend sind. So besteht ein Leuchtturm nicht nur aus dem Leuchtfeuer, sondern er hat auch Stockwerke mit ganz unterschiedlichen Funktionen. Zudem steht der Leuchtturm auf einem festen Fundament. Übertragen auf die Wirtschaft heißt das zweierlei:

(1) Auch im hoch entwickelten Industriestaat Deutschland können Lowtech-Unternehmen mit guten Produkten und Ideen erfolgreich sein. Ihre einfachen Produkte entstehen mit hoch technisierten Anlagen. In diesen Unternehmen arbeiten immer noch die meisten Beschäftigten in Deutschland (Hirsch-Kreinsen 2005).

(2) Problemlösungen zwischen Betrieb, Branche und Region sind dann Erfolg versprechend, wenn unterschiedliche Akteure an dem Prozess beteiligt sind und ein differenzierter Einsatz von Förderinstrumenten und -strategien organisiert werden kann. So ist die Zusammenarbeit in Unternehmensclustern dann besonders wirkungsvoll, wenn sie sich nicht nur auf die Anwendung neuer Technologien, sondern auch auf Weiterbildung, Qualifizierung und innovative Arbeitszeitgestaltung bezieht.

(3) Die Entwicklung von Zukunftsbranchen ist kein Selbstläufer. Sie muss in eine breite Strategie zur Wirtschaftsentwicklung eingebettet werden, die flankiert wird durch Bildung, Qualifizierung und Kriterien „Guter Arbeit“. Beschäftigte sind an einer sicheren, angemessen entlohnten und an einer guten Arbeit interessiert und daran, dass an ihrem Wohnort die Versorgung mit grundlegenden Dienstleistungen wie Bildung, Gesundheit und Handel gewährleistet ist.

\section{Schluss}

Trotz mancher positiver Ansätze ist die Wirtschafts- und Strukturpolitik aus beschäftigungspolitischer Sicht ambivalent zu beurteilen. Gerade in Krisenzeiten muss sie sich in erster Linie an der Sicherung und Schaffung von Arbeitsplätzen messen lassen und dies in quantitativer und qualitativer Hinsicht. Bislang ging die Politik davon aus, dass durch die Stärkung der Wettbewerbsfähigkeit von Unternehmen ganz automatisch mehr und bessere Arbeitsplätze entstehen und die bestehenden Arbeitsplätze sicherer werden. Diese Gesetzmäßigkeit ist seit einigen Jahren mit dem Entstehen von neuen Unternehmenskonzepten, der Dominanz des Shareholder-Value-Ansatzes und der kurzfristigen Renditeorientierung nicht mehr gültig. Ganz im Gegenteil: Die neue Strukturförderung unterstützt sogar den Wettbewerb um Unternehmen, Standorte und Fachkräfte. Staatliches Handeln wird rein nach betriebswirtschaftlichem Kostenkalkül bewertet, darüber hinausgehende gesellschaftspolitisch erstrebenswerte Ziele (Gerechtigkeit, Vollbeschäftigung, sozial-ökologisches Wirtschaften, flächendeckende Versorgung der Bevölkerung mit grundlegenden Dienstleistungen, existenzsicherndes Einkommen und vieles mehr) haben in dieser Strategie keinen Platz.

Nach wie vor werden in Deutschland massiv Unternehmen unterstützt. In der Regel werden die Subventionen an die $\mathrm{Zu}-$ sage von Arbeitsplätzen seitens des zu fördernden Unternehmens geknüpft. Wie die Förderpraxis aber zeigt, wird nicht selten die Zahl der zugesagten Arbeitsplätze nach unten korrigiert. Außerdem spielen bei der Mittelvergabe qualitative Aspekte der geförderten Arbeitsplätze keine Rolle. In den letzten - wirtschaftlich guten - Jahren haben sich die Arbeitsbedingungen der Beschäftigten vielfach verschlechtert. Heutein einer beschäftigungspolitisch schwierigen Zeit - erscheint es deshalb umso wichtiger, Kriterien ,guter Arbeit“ in die staatliche Förderpolitik aufzunehmen.

„Gute Arbeit" heißt in der Strukturpolitik, dass ein Unternehmen nur dann Sub- 
ventionen erhalten soll, wenn es Entgelte zahlt, die existenzsichernd sind, wenn es den Tarifvertrag einhält sowie Dauerarbeitsplätze schafft und erhält, die deutlich länger als fünf Jahre bestehen.

Um dieses zu erreichen, müssen Bund und Bundesländer in einem ersten Schritt eine transparente Förderpolitik umsetzen.
Einen guten Rahmen liefert hier die Europäische Transparenzinitiative (IP/06/ 1855 Brüssel, 20. Dezember 2006). Außerdem sollten die vorhandenen Beteiligungsstrukturen - insbesondere die, die im Rahmen der Europäischen Strukturfonds mit den Sozialpartnern aufgebaut wurden - für die Kontrolle genutzt werden. Jede Subven- tionierung eines Unternehmens sollte eine Innovationsvereinbarung enthalten, in der eine Strategie festgeschrieben wird, wie das Unternehmen besser werden will, sodass es zukünftig ohne Subvention wirtschaften und mehr und bessere Jobs anbieten kann.

\section{LITERATUR}

Blöcker, A. (2009): Mobile Module. Internationalisierung und Restrukturierung in der Autoindustrie, in: Ahlers, E./Kraemer, B./Ziegler, A.: Beschäftigte in der Globalisierungsfalle, Baden-Baden (im Erscheinen) Europäische Kommission (2008): Staatliche Beihilfen: Neuester Beihilfeanzeiger zeigt Trend zu gezielteren Maßnahmen, IP/08/1725, Brüssel 18. November

GEFRA/MR/TraSt (2004): Evaluierung des Einsatzes von Fördermitteln im Rahmen der Gemeinschaftsaufgabe "Verbesserung der regionalen Wirtschaftsstruktur" (GA) in den Jahren 1997-2003 in Thüringen, Münster Horn, G./Hohlfeld, P./Truger, A./Zwiener, R. (2009): Höheres Tempo erforderlich. Zu den Wirkungen des Konjunkturpakets II, IMK Policy Brief, Januar 2009

Institut für Makroökonomie und Konjunkturforschung (IMK) (2008):

Am Rande des Abgrunds, Prognose der wirtschaftlichen Lage 2009, IMK Report 35, Düsseldorf

Jakszentis, A./Hilpert, U. (2005): Regionale Entwicklungsunterschiede in Ostdeutschland im Vergleich. Differenzierungen in den neuen Bundesländern und Angleichungen an die alten Länder, Arbeitsheft 42, Frankfurt/Main

Kalmbach, P./Franke, R./Knottenbauer, K./Krämer, H. (2005): Die Interdependenz von Industrie und Dienstleistungen. Zur Dynamik eines komplexen Beziehungsgeflechts, Berlin

Koller, M. (2004): Wie erfolgreich sind Subventionen? - Investitionsförderung auf dem Prüfstand, IAB-Gutachten 1, Nürnberg

Hirsch-Kreinsen, H. (2005): „Low-Tech-Industrien“: Innovationsfähigkeit und Entwicklungschancen, in: WSI Mitteilungen 3, S. 144-150

Lichtblau, K./Ziegler, A. (1999): Die Metall- und Elektroindustrie in Deutschland. Aktuelle Situation und mittelfristige Perspektive der Beschäftigung, in: WSI-Mitteilungen 10, S. 694-698

Meißner, W./Fassing, W. (1989): Wirtschaftsstruktur und Strukturpolitik, München
Müller, G./Sträter, D. (2008): Raumplanung und regionale Förderpolitik Gleichwertige Lebensbedingungen im Wandel von Raumkonzeptionen und Leitbildern, in: Krumbein, W./Frieling von, H.-D./Kröcher, U./Sträter, D. (Hrsg.): Kritische Regionalwissenschaft. Gesellschaft, Politik, Raum, Münster

Rosenfeld, M./Franz, P./Günther, J./Heimpold, G./Kronthaler, F. (2006): Ökonomische Entwicklungskerne in ostdeutschen Regionen: Branchenschwerpunkte, Unternehmensnetzwerke und innovative Kompetenzfelder der Wirtschaft, in: IWH-Sonderhefte 5, Halle/Saale

Schalk, H. J./Untiedt G. (2000): Regional Investment Incentives in Germany: Impacts on Factor Demand and Growth, in: Annals of Regional Science 34, S. 173-195

Seifert, H. (2009): Kurzarbeit als Beschäftigungsbrücke, in: Böckler Impuls 2

Titze, M. (2007): Strategien der neuen Bundesländer im Rahmen der Gemeinschaftsaufgabe "Verbesserung der regionalen Wirtschaftsstruktur" Ein Vergleich, IWH-Diskussionspapiere 14

Wahse, J./Dahms, V. (2008): IAB-Betriebspanel Ost - Ergebnisse der zwölften Welle 2007, Berlin

Ziegler, A. (2003): Technologiepolitik und Mitbestimmung. Eine exemplarische Untersuchung in den Bundesländern Bayern, Nordrhein-Westfalen und Sachsen, Marburg

Ziegler, A. (2006): Strukturpolitik zwischen Ausgleich und Wachstum?, in: Schäfer C./Seifert H. (Hrsg.): Kein bisschen leise: 60 Jahre WSI, Hamburg, S. 325-334

Ziegler, A. (2007): Auf dem Weg zur Hightech-Road: die Innovationspolitik in Deutschland, in: Gerlach F./Ziegler A.: Innovationspolitik - wie kann Deutschland von anderen lernen?, Marburg, S. 176-201

Ziegler, A. (2008): Wirkungen von Standortverlagerung und Ausgliederung auf die Arbeitsbedingungen, in: WSI-Mitteilungen 6, S. 328-334 